IST-GDNL\#1/97

MIT-CTP\#2604

January 1997

hep-th/9704084

\title{
Stable Knotted Strings
}

\author{
Rui Dilão ${ }^{a} \dagger$ and Ricardo Schiappa $a_{\ddagger}^{b}$ \\ a- Grupo de Dinâmica Não-Linear, Departamento de Física \\ Instituto Superior Técnico, Av. Rovisco Pais \\ 1096 Lisboa Codex, Portugal \\ b- Center for Theoretical Physics and Department of Physics \\ Massachusetts Institute of Technology, 7 r Massachusetts Av. \\ Cambridge, MA 02139, U.S.A
}

\begin{abstract}
We solve the Cauchy problem for the relativistic closed string in Minkowski space $M^{3+1}$, including the cases where the initial data has a knot like topology. We give the general conditions for the world sheet of a closed knotted string to be a time periodic surface. In the particular case of zero initial string velocity the period of the world sheet is proportional to half the length $(\ell)$ of the initial string and a knotted string always collapses to a link for $t=\ell / 4$. Relativistic closed strings are dynamically evolving or pulsating structures in spacetime, and knotted or unknotted like structures remain stable over time. The generation of arbitrary $n$-fold knots, starting with an initial simple link configuration with non zero velocity is possible.
\end{abstract}

Phys. Lett. B 404 (1997) 57-65

Keywords: String Theory, Theoretical High Energy Physics.

PACS: $11.10 . \mathrm{Lm}$

$\dagger$ rui@sd.ist.utl.pt

$\ddagger$ ricardos@mit.edu 


\section{Introduction}

String theories and knot theory are playing a prominent role in modern theoretical physics, [1] and [2]. The foundations go back to Thomson's recognition of the invariance of vortex tubes in incompressible and non-viscous fluid flows and the hypothesis that atoms were knotted vortex tubes in ether [3]. In modern physics, string theory is becoming an important field of research due to the rich phenomena and physical concepts associated to nonlinear field theories in superstring theories [1], cosmic string theory [4-5], statistical mechanics [6], molecular biology [7] and ${ }^{3}$ He superfluids [8].

In the thirties Born and Infeld [9] proposed that the natural laws are independent of the spacetime coordinate systems and this should be expressed as the invariance of the action for any change of parameterization. Later, Nambu [10] proposed a relativistic string theory based on the invariance of the action for any parameterization of the world sheet of a string in Minkowski space. Therefore, the two-dimensional scalar equation of the BornInfeld nonlinear electrodynamics is the equation of motion of a string in the Minkowski

space $M^{2+1}$, [11], and the solution of this equation defines a surface of minimal area in $M^{2+1}$, the world sheet of a string.

The Cauchy problem for the equation of motion of an infinite relativistic string in $M^{2+1}$ has been solved [11]. The nonlinear nature of the solutions of this equation leads to the appearance of soliton like propagation, characterized by the coherence of wave packets before and after interaction, [12]. However, singularities arise when large amplitude travelling wave solutions collide [13]. Geometrically, these singularities correspond to the existence of folds and cusps in the integral surface associated to the solutions of the relativistic string equation, [12].

On the context of galaxy formation, Zeldovich suggested that strings formed in the early universe might provide the density perturbations needed to start the condensation process. Different grand unification phase transition temperatures derive from the string lifetimes and lengths. Kibble and Turok [4] have shown that any initially static closed string can self-intersect and collapse to a double loop, and conjectured that they can annihilate into particles. Hindmarsh and Kibble [5] have shown that for zero initial string velocity the world sheet of the string is a time periodic surface with period proportional to half the length of the initial string.

Besides the classical studies of solitons in strings, there has been a growing interest in string theory in the last few years, in the framework of quantum fields. In fact, the quantum study of solitonic solutions in superstring theory has become of central importance in modern string theory, as a nonperturbative probe into the strong coupling regime [14].

The Cauchy problem for open string configurations (free end points) appeared in the literature, [11], [15], [16] and [1]. The classical and quantum mechanics of a massless relativistic string with free end points moving at the speed of light has been analysed in 
[15]. However, as pointed out by Faddeev and Niemi [17], no explicit analysis of stable knotted structures exists for nonlinear field equations.

Here we solve the general Cauchy problem for the closed knotted string in $M^{3+1}$, showing that propagating knot like structures in Minkowski space $M^{3+1}$ are preserved, generating a time periodic world sheet. The singularity theory associated to string loop annihilation and creation is developed. In particular, we show that initially closed static strings always oscillate with the period $T=\ell / 2$, where $\ell$ is the string length. Knotted strings evolve to simple links with crossover, and initially closed simple links collapse to points. From points and simple links emerge closed knotted strings. The temporal periodicity of the string implies an infinite lifetime with periodic transitions from the simple link to the knotted string. We also show the existence of finite length string configurations that stretch indefinitely. Our analytical results are supplemented with simulations of the time evolution of the simple link, trefoil and figure eight knot.

\section{The Cauchy Problem for Knotted Strings}

We consider the Minkowsky space $M^{3+1}$ with metric $d \ell^{2}=d t^{2}-d x^{2}-d y^{2}-d z^{2}=$ $\left(d x^{0}\right)^{2}-\left(d x^{1}\right)^{2}-\left(d x^{2}\right)^{2}-\left(d x^{3}\right)^{2}$, and units such that $c=1$. A string $C$ in a general position in space at time $x^{0}=t$ is described by the set of spatial coordinates

$$
x^{k}(t, \sigma), \quad k=1,2,3
$$

where $\sigma$ is a parameter and $t$ is measured in the Lorentz frame of the string. We call $t$ the proper time of the string.

A propagating string $C$ in $M^{3+1}$ defines a two-dimensional surface $S$ with boundary $\partial S=C$, the world sheet of the string. The intersection of $S$ with the hyperplane $x^{0}=t$ is the configuration of the string at time $t$.

In general, in a metric space, the first fundamental form of a surface is a quadratic form $g_{i j} d x^{i} d x^{j}$, and the infinitesimal area of a surface is proportional to the square root of the determinant of the covariant tensor $g_{i j}$. Nambu [10] postulated that the action for the motion of the string in Minkowski space is proportional to the area of its world sheet $S$,

$$
A=-m \iint d p d q \sqrt{\left(\partial_{p} \vec{r} \cdot \partial_{q} \vec{r}\right)^{2}-\left(\partial_{p} \vec{r}\right)^{2}\left(\partial_{q} \vec{r}\right)^{2}}
$$

where $\vec{r}=\left(x^{0}(p, q), x^{1}(p, q), x^{2}(p, q), x^{3}(p, q)\right), p$ and $q$ are the parameters of the surface $S$ and $m$ is a constant of proportionality with dimension of (length) ${ }^{-2}$. As the area of a surface, $\iint \sqrt{g} d p d q$, is independent of the parameterization, it is proportional to the action (2.2).

For a string in $M^{3+1}$ it is natural to take $p=t$ and $q=\sigma$, but in general $p$ and $q$ are functions of $t$ and $\sigma$. In the following we adopt this more general viewpoint. 
The Euler-Lagrange equations for the motion of the string in spacetime are derived from the action $(2.2)$, where the Lagrangian is $\mathcal{L}=-m \sqrt{\left(\partial_{p} \vec{r} \cdot \partial_{q} \vec{r}\right)^{2}-\left(\partial_{p} \vec{r}\right)^{2}\left(\partial_{q} \vec{r}\right)^{2}}$. However, with the choice $p=t$ and $q=\sigma$ we obtain three second order nonlinear partial differential equations that are difficult to analyse. Instead, we take the arbitrary parameterization $p=p(t, \sigma)$ and $q=q(t, \sigma)$ and we simplify the action with a trick introduced by Barbashov and Chernikov [11]. These authors have shown that the hyperbolicity of the Euler-Lagrange equations implies a choice of the parameters $p$ and $q$ in such a way that

$$
\left(\partial_{p} \vec{r}\right)^{2}=0, \quad\left(\partial_{q} \vec{r}\right)^{2}=0
$$

Under these conditions, the action (2.2) simplifies,

$$
A=-m \iint d p d q\left(\partial_{p} \vec{r} \cdot \partial_{q} \vec{r}\right)
$$

With the new parameters $p$ and $q$ obeying the constraint equations (2.3) and to be determined later, the Euler-Lagrange equation of motion for the free string in Minkowski space is

$$
\partial_{p} \partial_{q} \vec{r}=0
$$

Equation (2.5) is readily solved and the general solution is

$$
x^{\mu}(p, q)=f^{\mu}(p)+g^{\mu}(q), \quad \mu=0,1,2,3
$$

where $f^{\mu}$ and $g^{\mu}$ are arbitrary functions that must be determined from initial data and the constraint equations (2.3). In order to determine $f^{\mu}$ and $g^{\mu}$ from initial data and constraint equations - Cauchy problem - , we express the solutions (2.6) in the form $(2.1)$,

$$
x^{\mu}(t, \sigma)=x^{\mu}(p(t, \sigma), q(t, \sigma)), \quad \mu=0,1,2,3
$$

At $t=0$, the initial position and velocity of a string is specified parametrically by the functions

$$
\begin{aligned}
x^{k}(0, \sigma) & =a^{k}(\sigma) \\
\left.\partial_{t} x^{k}(t, \sigma)\right|_{t=0} & =b^{k}(\sigma), \quad k=1,2,3
\end{aligned}
$$

If the $a^{k}(\sigma)$ and $b^{k}(\sigma)$ are periodic functions in $\sigma$, these functions describe a closed curve (knotted or unknotted) in the subspace $t=0$ of $M^{3+1}$. Without loss of generality, we take the periodicity conditions $a^{k}(\sigma)=a^{k}(\sigma+2 \pi)$ and $b^{k}(\sigma)=b^{k}(\sigma+2 \pi)$, where $2 \pi$ is the least period for the functions $a^{k}(\sigma)$. Initial string velocities can have least period $2 \pi / n$, where $n$ is some positive integer.

As the parameters $p$ and $q$ are arbitrary, the constraint equations (2.3) do not change if we take $p=h_{1}\left(p^{\prime}\right)$ and $q=h_{2}\left(q^{\prime}\right)$, with $\left(\partial_{p^{\prime}} h_{1}\right) \neq 0$ and $\left(\partial_{q^{\prime}} h_{2}\right) \neq 0$. Therefore, for 
$t=0$, we choose $p=q=\sigma\left(p^{\prime}=q^{\prime}=\sigma\right.$, and $h_{1}$ and $h_{2}$ are the identity, for $\left.t=0\right)$. Under these conditions, comparing (2.8) with (2.6), we have, for $t=0$,

$$
\begin{aligned}
& \left.x^{0}(p, q)\right|_{p=q}=\left.t(p, q)\right|_{p=q}=f^{0}(\sigma)+g^{0}(\sigma)=0 \\
& \left.x^{k}(p, q)\right|_{p=q}=f^{k}(\sigma)+g^{k}(\sigma)=a^{k}(\sigma), \quad k=1,2,3
\end{aligned}
$$

Writing the solution (2.6) in d'Alembertian form

$$
\vec{r}(p, q)=\frac{1}{2}(\vec{\rho}(p)+\vec{\rho}(q))+\frac{1}{2} \int_{p}^{q} \vec{\pi}(s) d s
$$

and comparing (2.10) with (2.9) for $t=0$, we conclude that $\left.\vec{\rho}(p)\right|_{t=0}=\left(0, a^{1}(\sigma), a^{2}(\sigma), a^{3}(\sigma)\right)$. So, the solution $(2.6)$ can now be written as

$$
\begin{aligned}
t(p, q) & =\frac{1}{2} \int_{p}^{q} \pi^{0}(s) d s \\
x^{k}(p, q) & =\frac{1}{2}\left(a^{k}(p)+a^{k}(q)\right)+\frac{1}{2} \int_{p}^{q} \pi^{k}(s) d s, \quad k=1,2,3
\end{aligned}
$$

where $\pi^{\mu}$ are four unknown functions. Calculating the derivatives $\partial_{p} x^{k}$ and $\partial_{q} x^{k}$ from (2.11), with $p$ and $q$ as functions of $t$ and $\sigma$, and solving for $\partial_{t} x^{k}$ and $\partial_{\sigma} x^{k}$ at $t=0$, $p=q,\left.\partial_{p} \sigma\right|_{t=0}=\left.\partial_{q} \sigma\right|_{t=0}$, and $\left.\partial_{t} x^{k}\right|_{t=0}=\pi^{k}(\sigma) / \pi^{0}(\sigma)=b^{k}(\sigma)$. Therefore, $\pi^{k}=b^{k} \pi^{0}$ are directly computed from initial velocities (2.8) and $\pi^{0}$. For $t=0$, the constraint equations (2.3) become

$$
\begin{aligned}
& \left(\pi^{0}\right)^{2}=\left(\partial_{\sigma} a^{1}-b^{1} \pi^{0}\right)^{2}+\left(\partial_{\sigma} a^{2}-b^{2} \pi^{0}\right)^{2}+\left(\partial_{\sigma} a^{3}-b^{3} \pi^{0}\right)^{2} \\
& \left(\pi^{0}\right)^{2}=\left(\partial_{\sigma} a^{1}+b^{1} \pi^{0}\right)^{2}+\left(\partial_{\sigma} a^{2}+b^{2} \pi^{0}\right)^{2}+\left(\partial_{\sigma} a^{3}+b^{3} \pi^{0}\right)^{2}
\end{aligned}
$$

After developing the right hand side of (2.12), we finally obtain,

$$
\begin{aligned}
& \left(\pi^{0}\right)^{2}\left(1-\sum_{i=1}^{3}\left(b^{i}\right)^{2}\right)+2 \pi^{0}\left(\sum_{i=1}^{3} b^{i} \partial_{\sigma} a^{i}\right)-\left(\sum_{i=1}^{3}\left(\partial_{\sigma} a^{i}\right)^{2}\right)=0 \\
& \left(\pi^{0}\right)^{2}\left(1-\sum_{i=1}^{3}\left(b^{i}\right)^{2}\right)-2 \pi^{0}\left(\sum_{i=1}^{3} b^{i} \partial_{\sigma} a^{i}\right)-\left(\sum_{i=1}^{3}\left(\partial_{\sigma} a^{i}\right)^{2}\right)=0
\end{aligned}
$$

Solving the above equations, we obtain the following solution, compatible with the $p, q$-parameterization:

$$
\pi^{0}(\sigma)= \pm\left(\frac{\sum_{i=1}^{3}\left(\partial_{\sigma} a^{i}(\sigma)\right)^{2}}{1-\sum_{i=1}^{3}\left(b^{i}(\sigma)\right)^{2}}\right)^{1 / 2}
$$


provided $0 \leq \sum_{i=1}^{3}\left(b^{i}(\sigma)\right)^{2}<1$ and $\sum_{i=1}^{3} b^{i}(\sigma) \partial_{\sigma} a^{i}(\sigma)=0$.

With, $\pi^{k}(\sigma)=b^{k}(\sigma) \pi^{0}(\sigma)$ and introducing (2.14) into (2.11), the forward time solution of equation $(2.5)$ is

$$
\begin{aligned}
t(p, q) & =\frac{1}{2} \int_{p}^{q}\left(\frac{\sum_{i=1}^{3}\left(\partial_{s} a^{i}(s)\right)^{2}}{1-\sum_{i=1}^{3}\left(b^{i}(s)\right)^{2}}\right)^{1 / 2} d s \\
x^{k}(p, q) & =\frac{1}{2}\left(a^{k}(p)+a^{k}(q)\right)+\frac{1}{2} \int_{p}^{q} b^{k}(s)\left(\frac{\sum_{i=1}^{3}\left(\partial_{s} a^{i}(s)\right)^{2}}{1-\sum_{i=1}^{3}\left(b^{i}(s)\right)^{2}}\right)^{1 / 2} d s, k=1,2,3
\end{aligned}
$$

provided $0 \leq \sum_{i=1}^{3}\left(b^{i}(\sigma)\right)^{2}<1, \sum_{i=1}^{3} b^{i}(\sigma) \partial_{\sigma} a^{i}(\sigma)=0$ and $p=q=\sigma$, for $t=0$. In the particular case of zero initial velocities, $b^{k}(\sigma)=0$, for $k=1,2,3$, we have

$$
\begin{aligned}
t(p, q) & =\frac{1}{2} \int_{p}^{q}\left(\sum_{i=1}^{3}\left(\partial_{s} a^{i}(s)\right)^{2}\right)^{1 / 2} d s \\
x^{k}(p, q) & =\frac{1}{2}\left(a^{k}(p)+a^{k}(q)\right), k=1,2,3
\end{aligned}
$$

Note that, if we take $q \geq p$, the choice of the plus sign in (2.14) is equivalent to say that the solution (2.15) is forward in time. If we let $q<p$ in (2.15), we obtain a solution backwards in time, that could also be calculated with the choice of the minus sign in (2.14) with $q \geq p$. Therefore, with $p, q \in \mathbf{R}$, the choice of the plus or minus sign in (2.14) leads to the same solution (2.15). It can be straightforwardly shown that this reversibility property of the solutions (2.15) are derived from the nonlinear equations of motion in the $t$ and $\sigma$ coordinates, directly derived from the action (2.2) with $p=t$ and $q=\sigma$.

The condition $0 \leq \sum_{i=1}^{3}\left(b^{i}(\sigma)\right)^{2}<1$ on the initial data imposes the constraint that the transverse string velocity is always below 1 . (Note that according to our choice of units $c=1)$. The second condition, $\sum_{i=1}^{3} b^{i}(\sigma) \partial_{\sigma} a^{i}(\sigma)=0$, is a transversal constraint between the initial velocity and the parametric representation of the string, and can be written in the form $\left.\left(\partial_{t} \vec{r} \cdot \partial_{\sigma} \vec{r}\right)\right|_{t=0}=0$. Physically, this means that the solution (2.16) exists as far as strings are not allowed to break.

Hence, the Cauchy problem for a closed knotted string in $M^{3+1}$ is solved.

From the above solution we derive now an important property for the topology of the world sheet of the string. Let a closed string be parameterized at $t=0$ by periodic functions $a^{k}(\sigma)$, with $k=1,2,3$, and $a^{k}(\sigma+2 \pi)=a^{k}(\sigma)$, with $\sigma \in \mathbf{R}$. By construction, $b^{k}(\sigma)$ is also periodic with period $2 \pi / n$, where $n$ is a positive integer. By (2.15), it follows that

$$
x^{k}(p, q+2 \pi)=x^{k}(p, q)+\frac{1}{2} \int_{q}^{q+2 \pi} b^{k}(s)\left(\frac{\sum_{i=1}^{3}\left(\partial_{s} a^{i}(s)\right)^{2}}{1-\sum_{i=1}^{3}\left(b^{i}(s)\right)^{2}}\right)^{1 / 2} d s, k=1,2,3
$$


As the integrand in the above equation has least period $2 \pi$, and if the functions $b^{k}(\sigma)$ are such that the three integrals in (2.17) are zero, we have $x^{k}(p, q+2 \pi)=x^{k}(p, q)$ and, therefore, the world sheet $S$ is periodic in the spatial coordinates $x^{k}$. To determine the proper time period of the world sheet, we have, by (2.15),

$$
t(p, q+2 \pi)=t(p, q)+\frac{1}{2} \int_{q}^{q+2 \pi}\left(\frac{\sum_{i=1}^{3}\left(\partial_{s} a^{i}(s)\right)^{2}}{1-\sum_{i=1}^{3}\left(b^{i}(s)\right)^{2}}\right)^{1 / 2} d s:=t(p, q)+T
$$

where $T$ is the period of the world sheet. In the particular case where $b^{k}(\sigma)=0$, for $k=1,2,3$, we obtain $T=\ell / 2$, where $\ell$ is the length of the string at $t=0$, [5]. (Note that $c=1$. In SI units we have $T=\ell /(2 c))$. So, zero initial velocity closed strings pulsate in spacetime. If some $b^{i}(\sigma) \neq 0$ and the integral in (2.17) is zero, the period is given by the integral in (2.18).

If the integral in (2.17) is non zero, then, this integral as a function of $q$, will be unbounded with increasing $q$ and the world sheet of the string is no longer periodic in time. As $t(p, q)$ is an increasing function of $q$, in the limit $t \rightarrow \infty, x^{k} \rightarrow \pm \infty$. In this case, the string will become infinitely long as proper time passes.

\section{Singularities and Knotted String Solutions}

To depict the configuration of a string in $M^{3+1}$ at time $t$, with zero initial velocities, we solve the implicit equation (2.16a). So, for fixed $p=p_{0}$ and $t=t_{0}$, the value of $q$ is the root of the equation

$$
\frac{1}{2} \int_{p_{0}}^{q}\left(\sum_{i=1}^{3}\left(\partial_{s} a^{i}(s)\right)^{2}\right)^{1 / 2} d s-t_{0}=0
$$

Let $q_{0}$ be a root of (3.1). Therefore, if $q_{00}$ is an initial guess for the value of $q_{0}$, by the Newton method,

$$
q_{0 n+1}=q_{0 n}-2 \frac{t\left(p_{0}, q_{0 n}\right)-t_{0}}{\left(\sum_{i=1}^{3}\left(\left.\partial_{s} a^{i}(s)\right|_{s=q_{0 n}}\right)^{2}\right)^{1 / 2}}
$$

and in the limit $n \rightarrow \infty, q_{0 n} \rightarrow q_{0}$. Therefore, for a given $t_{0}$ and $p_{0}$ we determine $q_{0}$ by (3.2), and the string configuration is given by (2.16b). The general case with $b^{k} \neq 0$ is analogously derived from (2.15).

In Fig. 1, we show the world sheet of the simple link in the Minkowski space $M^{2+1}$, for zero initial velocity. In Figs. 2 and 3, the trefoil and figure eight knot configurations at successive times are shown, for initially static strings. In these cases, the world sheets are time periodic surfaces, as seen in $\S 2$. However, in these simulations, there exists a time $t=t^{\prime}$, with $0<t^{\prime}<T$, for which the string loop self intersects, leading to the loss of injectivity of the map $(p, q) \rightarrow S \in M^{3+1}$, defined by (2.15). Under these circumstances, 
we say that the string develops a singularity. Also, this singularity depends on the type of knot we choose as initial configuration. As seen in Fig. 1, 2 and 3, the simple link collapses to a point, while both the trefoil and figure eight knot collapse to a simple link.

In order to analyse the string singularities during time evolution, we introduce the derivative $D$ of the map $(p, q) \rightarrow S$. By $(2.11)$,

$$
D=\left(\begin{array}{cc}
\partial_{p} t & \partial_{q} t \\
\partial_{p} x^{1} & \partial_{q} x^{1} \\
\partial_{p} x^{2} & \partial_{q} x^{2} \\
\partial_{p} x^{3} & \partial_{q} x^{3}
\end{array}\right)=\frac{1}{2}\left(\begin{array}{cc}
-\pi^{0}(p) & \pi^{0}(q) \\
\partial_{p} a^{1}(p)-\pi^{1}(p) & \partial_{q} a^{1}(q)+\pi^{1}(q) \\
\partial_{p} a^{2}(p)-\pi^{2}(p) & \partial_{q} a^{2}(q)+\pi^{2}(q) \\
\partial_{p} a^{3}(p)-\pi^{3}(p) & \partial_{q} a^{3}(q)+\pi^{3}(q)
\end{array}\right)
$$

and the point $\vec{r}(p, q) \in S$ is nonsingular if the rank of $D$ is 2 . The singularities on the world sheet $S$ occur for $p$ and $q$ values where the rank of $D$ is 1 . So, as the row and column ranks of a matrix are equal, the matrix $\mathrm{D}$ has rank 1 if any two row vectors of $D$ are linearly dependent. Therefore, let $\alpha$ and $\beta$ be constants such that $\alpha\left(-\pi^{0}(p), \pi^{0}(q)\right)+\beta\left(\partial_{p} a^{k}(p)-\right.$ $\left.\pi^{k}(p), \partial_{q} a^{k}(q)+\pi^{k}(q)\right)=0$. Solving this equation for $\alpha$ and $\beta$, it follows that any two row vectors of $D$ are linearly dependent if $p$ and $q$ are simultaneous solutions of the equations

$$
\pi^{0}(q)\left(\partial_{p} a^{k}(p)-\pi^{k}(p)\right)+\pi^{0}(p)\left(\partial_{q} a^{k}(q)+\pi^{k}(q)\right)=0, k=1,2,3
$$

Let us now analyse the singularities of the simple link, trefoil and figure eight knot, in the cases of Figs. 1, 2 and 3.

For the simple link of Fig. 1, we have $a^{1}(\sigma)=\cos (\sigma), a^{2}(\sigma)=\sin (\sigma)$ and $b^{k}(\sigma)=0$, for $k=1,2$. As $\pi^{k}=0$, for $k=1,2$, by (3.3), the singular values of the derivative $D$, in the $p$ and $q$ plane, is the set of points $(p, q)$ that are simultaneous solutions of the equations $-\sin (p)-\sin (q)=0$ and $\cos (p)+\cos (q)=0$. Hence, these singular values lie on the graphs of the curves $q=p \pm \pi+2 \pi n$ in the $(p, q)$ plane, where $n$ ranges over all the integers. Introducing the relation $q=p \pm \pi+2 \pi n$ into (2.16a), for this particular case of initial data, it follows that singularities do occur for $t=(2 n+1) \ell / 4$, where $\ell=2 \pi$ is the length of the string at time $t=0$, Fig. 1. Introducing $(p, q)=(p, p \pm \pi+2 \pi n)$ into (2.16b), we obtain $x^{k}(p, q)=0$ for all $p, q \in \mathbf{R}$. Therefore, for $t=(2 n+1) \ell / 4$, the string collapses into a point. Outside these singularities, the simple link configuration is stable, evolving in spacetime $M^{2+1}$ as a pulsating structure.

The singularity analysis for the trefoil knot is more evolved. In Fig. 2 we took the initial parameterization $a^{1}(\sigma)=(2.0+0.6 \cos (3 \sigma)) \cos (2 \sigma), a^{2}(\sigma)=(2.0+0.6 \cos (3 \sigma)) \sin (2 \sigma)$, $a^{3}(\sigma)=2 \sin (3 \sigma)$ and $b^{k}(\sigma)=0$, for $k=1,2,3$. Singular solutions of the equations of motion occur at the triple intersections of the zero level set of the three functions in (3.3), Fig. 4a). In this case, numerical analysis shows that the singularity occurs for $t=\ell / 4$, where, in this example, $\ell=37.1587$. At the singular points the trefoil knot configuration of the string collapses to a simple link. Outside the singularities, the trefoil knot is stable. 
In the figure eight knot, Fig. 3, the periodicity of the world sheet is also $T=\ell / 2$, but singular solutions occur for several proper time values between 0 and $\ell / 2$. In this parameterization, in each half period of the world sheet there exist several time values for which the knot folds cross each other, unknotting and knotting the closed string during time evolution. In this case, the zero level sets of the three functions in (3.3) are presented in Fig. 4b). Observe however that for $t=\ell / 4=4.45423$, the singular solution corresponds to the simple link. Outside these singularities, the figure eight knot is stable.

The existence of singularities at $t=\ell / 4$ in the examples of Fig. 1-3, lead to the conjecture that every knot configuration of an initially static string will cross the simple link configuration, independently of the initial parameterization. This $t=\ell / 4$ collapse to a link can then be seen as a universal property for knotted strings.

Due to the reversibility property of the solution (2.15), we can have a simple link as initial configuration and obtain a $n$-fold knot. This is easily obtained with nonzero initial velocities.

\section{Conclusions}

We have solved explicitly the Cauchy problem for closed knotted strings in Minkowski space $M^{3+1}$. We have shown that the world sheet of a closed string can be periodic in time or can stretch infinitely as $t \rightarrow \infty$. The period has been calculated and is related with the string dimensions. Strings are always stable structures in spacetime. Due to the reversibility property of the equations of motion, the dynamical generation of $n$-fold knotted strings from simple links is possible.

Future work in exact solutions of string theories include the study of (self-gravitating) knotted strings in a Friedman-Robertson-Walker spacetime and quantization of knotted strings. Important questions arising from this work concern the effect of singularities on string diagram calculus, [1], and changes in the partition function due to knotted strings $[18]$.

Acknowledgments: We would like to thank Kenneth Johnson for a careful reading of the manuscript. One of us (RS) is partially supported by the Praxis XXI grant BD3372/94 (Portugal). 


\section{References}

[1] - M. B. Green, J. H. Schwarz \& E. Witten, Superstring Theory, Cambridge Univ. Press (1987).

[2] — L. H. Kauffman, Knots and Physics, World-Scientific (1991).

[3] - W. Thomson, On Vortex Motion, Trans. R. Soc. Edinburgh XXV (1869) 217-260.

[4] — T. W. B. Kibble \& N. Turok Self-Intersection of Cosmic Strings, Phys. Lett. $116 B$ (1982) 141-143.

[5] — M. B. Hindmarsh \& T. W. B. Kibble, Cosmic Strings, Rep. Prog. Phys. 58 (1995) 477, hep-ph/9411342.

[6] - V. F. R. Jones, On Knot Invariants Related to some Statistical Mechanics Models, Pacific J. of Math. 137 (1989) 311-334.

[7] — D. W. L. Sumners, Knot Theory and DNA, Proc of Symposia in App. Math. 45 (1991) 39-72.

[8] — C. Bäuerle, Yu. M. Bunkov, S. N. Fisher, H. Godfrin \& G. R. Pickett, Laboratory Simulation of Cosmic String Formation in the Early Universe Using Superfluid ${ }^{3}$ He, Nature 382 (1995) 332-334.

[9] — M. Born \& L. Infeld, Foundations of the New Field Theory, Proc. Roy. Soc. A144 (1934) 425-451.

[10] - Y. Nambu, Lectures at the Copenhagen Summer Symposium (1970).

[11] — B. M. Barbashov \& N. A. Chernikov, Solution and Quantization of a Nonlinear Two-Dimensional Model for a Born-Infield Type Field, Soviet Phys. JETP 23 (1966) 861868.

[12] — S. Brundobler \& V. Elser, Colliding Waves on a Relativistic String, Am. J. Phys. 60 (1992) 726-732.

[13] - B. M. Barbashov \& N. A. Chernikov, Solution of the Two Plane Wave Scattering Problem in a Nonlinear Scalar Field Theory of the Born-Infield Type, Soviet Phys. JETP 24 (1967) 437-442.

[14] — M. J. Duff, R. R. Khuri \& J. X. Lu, String Solitons, Phys. Rept. 259 (1995) 213, hep-th/9412184 (1994).

[15] — P. Goddard, J. Goldstone, C. Rebbi \& C. B. Thorn, Quantum Dynamics of a Massless Relativistic String, Nuc. Phys. B56 (1973) 109-135.

[16] - J. Scherk, An Introduction to the Theory of Dual Models and Strings, Rev. Mod. Phys. 47 (1975) 123-164.

[17] — L. Faddeev \& A. J. Niemi, Stable Knot-Like Structures in Classical Field Theory, Nature 387 (1997) 58-61, hep-th/9610193 (1996).

[18] - M. Zaganescu, Bosonic Knotted Strings and Cobordism Theory, Europhys. Lett. 4 (1987) 521-525. 


\section{Figure Captions}

Figure 1: Oscillations of the simple link in $M^{2+1}$, calculated from (2.16), for the initial configuration $a^{1}(\sigma)=\cos (\sigma), a^{2}(\sigma)=\sin (\sigma)$ and $b^{k}(\sigma)=0$, for $k=1,2$. Due to the singularities occurring for $t=\ell / 4,3 \ell / 4, \ldots$, where $\ell=2 \pi$ is the initial configuration length, the circle collapses into a point. The world sheet $S$ displayed has parametric representation $(\cos (t) \cos (p-t), \cos (t) \sin (p-t), t)$, derived directly from $(2.16)$.

Figure 2: Time evolution of the trefoil knot in $M^{3+1}$, for several values of $t$ and calculated from (2.16). We represent a tubular neighborhood around the string in order to better depict the knot topology. The world sheet is periodic along the $t$ axis with period $T=\ell / 2=18.5942$. For $T=\ell / 4=9.2971$, the trefoil loops collapse and a singularity appears. Singularities occur for $t=(2 n+1) \ell / 4$, where $\ell$ is the string initial length. Due to time parity, the string evolution in the time interval $\ell / 4 \leq t \leq \ell / 2$ follows the reverse order of the time evolution in the interval $0 \leq t \leq \ell / 4$.

Figure 3: Time evolved configuration of the figure eight knot and creation of singularities in the time interval $0 \leq t \leq \ell / 4$.

Figure 4: Zero level set of the functions in (3.3), for the trefoil and figure eight knot. The dots are the values in the $(p, q)$ plane where singularities of the trefoil knot of Fig. 2 (a) and figure eight knot of Fig. 3 (b) occur. The singular $(p, q)$-values correspond to the triple intersection of the level lines. These singularity diagrams are parameterization dependent and correspond to a simple qualitative analysis of (3.3). 
This figure "fig1.gif" is available in "gif" format from: http://arXiv.org/ps/hep-th/9704084v3 
This figure "fig2.gif" is available in "gif" format from: http://arXiv.org/ps/hep-th/9704084v3 
This figure "fig3.gif" is available in "gif" format from: http://arXiv.org/ps/hep-th/9704084v3 
This figure "fig4.gif" is available in "gif" format from: http://arXiv.org/ps/hep-th/9704084v3 